\title{
LA FELICIDAD COMO PREDICTOR DEL RENDIMIENTO ACADÉMICO DE LOS ESTUDIANTES UNIVERSITARIOS. UN ANÁLISIS COMPARATIVO ENTRE MÉXICO Y ESPAÑA
}

\author{
HAPPINESS AS A PREDICTOR OF ACADEMIC PERFORMANCE IN \\ UNIVERSITY STUDENTS. A COMPARATIVE ANALYSIS BETWEEN \\ MEXICO AND SPAIN
}

\author{
RAFAEL RAVINA-RIPOLL \\ Universidad de Cádiz \\ EDUARDO AHUMADA-TELLO* \\ Universidad Autónoma de Baja California \\ E. JULIÁN GÁLVEZ-AlBARRACÍN \\ Universidad del Valle
}

Recibido: 05/05/2019 Aceptado: 19/06/2019

\section{RESUMEN}

Durante las dos primeras décadas del siglo XXI, la investigación científica sobre la felicidad cognitiva en las aulas de las instituciones de educación superior se ha intensificado, algunas de ellas se han utilizado para explorar en el marco de la sociedad digital si la promoción del aprendizaje colaborativo es una Estrategia didáctica útil para estimular el nivel de felicidad de los futuros egresados. Coherentemente con este enfoque de investigación, el presente trabajo tiene como objetivo analizar el vínculo entre la felicidad de los estudiantes universitarios y su rendimiento académico. Para este propósito, durante el año académico 2018-2019, se tomó una muestra de estudiantes de tecnología,

\footnotetext{
* Autor de correspondencia: eahumada@uabc.edu.mx
} 
administración, contabilidad y negocios internacionales de las universidades de Cádiz (España) y la Autónoma de Baja California (México). Los resultados obtenidos por regresiones lineales son bastante consistentes con la literatura científica sobre la correlación entre la felicidad y un mejor rendimiento académico, ambas poblaciones confirman esta teoría.

Palabras clave: Felicidad, rendimiento académico, universidad, sociedad digital.

\section{ABSTRACT}

During the first two decades of the 21 st century, the scientific research about cognitive happiness within the classrooms of higher education institutions have been intensified, some of them have been used to explore within the framework of the digital society whether the promotion of collaborative learning is a useful didactic strategy to stimulate the happiness level of future graduates. Coherently with this research focus, the present work aims to analyze the link between happiness of university students and their academic performance. For this intention, during the academic year 2018-2019, a sample was taken from students of technology, administration, accounting and international business from the universities of Cádiz (Spain) and the Autonomous of Baja California (Mexico). The results obtained by linear regressions are quite consistent with the scientific literature about the correlation between happiness and a better academic performance, both populations confirm this theory.

Keywords: Happiness, academic performance, university, digital society.

\section{INTRODUCCIÓN}

Actualmente, un volumen importante de las universidades que se sitúan en los puestos altos de los rankings de excelencia académica y competitividad se caracterizan por tener un sólido modelo de aprendizaje de tipo colaborativo y adaptativo que ayuda a enriquecer los conocimientos digitales y las competencias transversales de sus estudiantes (Kindelán 2013). En un mercado laboral donde los avances tecnológicos y la automatización de los procesos productivos demandan cada día más a personas con alta cualificación profesional y elevadas habilidades sociales y de comunicación. Ante esta realidad, las principales autoridades de las instituciones de enseñanza superior deben impulsar un método de enseñanza inclusivo destinado a incrementar el razonamiento empírico, la inteligencia emocional, el debate aristotélico, la felicidad, el espíritu crítico, la calidad de vida y el humanismo de los futuros graduados (Pérez Muñoz y 
Castaño Calle, 2016). De ahí la necesidad de cultivar un semillero de aulas que se conviertan en espacios dinámicos y abiertos hacia el diálogo colectivo, la imaginación, el aprendizaje autónomo, la cocreación colaborativa y el trabajo en equipo. Esto permite a los alumnos poder participar activamente en la construcción de sus conocimientos cognitivos y aptitudes sociales, mediante un estilo docente positivo orientado a fomentar la tolerancia, el bien común, la motivación, el emprendimiento, la empatía o la investigación científica. (Martínez, Meneghel y Peñalver 2019).

De esta manera se podrá acabar con los modelos educativos de tipo teórico y repetitivo, los cuales limitan desarrollar una didáctica disruptiva, creativa y adaptativa. Bajo estos principios rectores, hay que señalar que las prestigiosas universidades de Harvard y Stanford están llevando a cabo una importante reforma pedagógica de sus planes docentes, con la finalidad de poder implementar en todas sus titulaciones académicas, el círculo virtuoso de la innovación y el currículo inteligente 1.0 en la era de la Industria 4.0 (Flores 2018). Para ello se precisa, entre otras cosas, de docentes innovadores, colaborativos y abiertos a la participación libre y creativa de los alumnos a través de una enseñanza que contribuya positivamente a la búsqueda holística de su felicidad. Respecto a esta última cuestión, existe una escasa producción bibliográfica que demuestra que los educandos felices presentan altas cotas de rendimiento académico en el contexto universitario. Desde esta perspectiva, el artículo que aquí se presenta con el título "La felicidad como predictor del rendimiento académico de los estudiantes universitarios en la sociedad digital. Un análisis comparativo entre México y España"; tiene finalidad explorar el constructo felicidad- rendimiento académico en los estudiantes de las universidades de Cádiz (España) y de la Autónoma de Baja California (México) durante parte del ciclo académico 20182019. Adicionalmente, los resultados alcanzados por dicho estudio pueden ser de gran utilidad para los rectores y su equipo de gobierno en el diseño de futuras políticas educativas destinadas a mejorar el bienestar subjetivo de sus alumnos en la sociedad del conocimiento (Ahumada- Tello, Hernández G de Velazco y Ravina-Ripoll 2018).

El trabajo se estructura de la siguiente manera. En el primer apartado se ilustra la revisión literaria que sirve de base para el objeto de nuestra investigación. En el segundo apartado se describe la metodología utilizada, así como la definición de las muestras sujeto del estudio, en este caso estudiantes mexicanos y españoles. En los tres siguientes apartados se exponen brevemente la metodología, la escala de medición de las variables aplicadas al análisis empírico, así como el modelo de regresión utilizado para determinar la incidencia que posee el parámetro felicidad en el rendimiento académico respectivamente. A 
continuación, se muestra los resultados obtenidos, para finalmente mostrar las conclusiones más relevantes llegadas por este artículo.

\section{MARCO TEÓRICO}

Dentro de la disciplina de las ciencias sociales la bibliografía científica que estudia la felicidad académica es bastante rica y amplia desde principios del presente siglo, especialmente en el área de la educación, la psicología y la economía (Öztemel y Yildiz-Akyol 2019; Sezer y Can 2019; Jongbloed 2018; Rojas 2016; Flynn y MacLeod 2015; Martin 2011; Chan, Miller y Tcha 2005; Noddings 2003). Muchas de las investigaciones efectuadas durante dicho periodo entienden la felicidad como sinónimo de los términos bienestar subjetivo o satisfacción con la vida (Charles-Leija, Aboites, y Llamas 2018; Veenhoven 2015; Alarcón 2006; Lyubomirsky, Tkach y DiMatteo 2006); lo que favoreció la aparición de múltiples investigaciones destinadas a explorar empíricamente si el nivel de educación alcanzados por las personas tiene un impacto positivo y directo en su felicidad (Easterbrook, Kuppens y Manstead 2016; Nikolaev y Rusakov 2016; Frey y Stutzer 2002).

De otra parte Álvarez-Pertuz et.al. (2017) manifiestan que la educación es un proceso continuado que busca potencializar al ser humano para lograr mayor integralidad. En relación con ello autores como Paso-Niebla y Hernández-Guzmán (2007) y Del Valle y Urquijo (2015) señalan que el rendimiento académico es el grado en el que un individuo logra los objetivos de aptitudes y conocimientos establecidos para un programa de formación determinado, lo cual al mismo tiempo tiene implicaciones en el nivel de bienestar y felicidad de dicha persona (Paschoal, Torres y Barreiros 2010; Posada y Aguilar 2012; Moreno y Marrero 2015).

No obstante a lo señalado en el párrafo anterior, muchas de las universidades siguen implementando un modelo de aprendizaje que pone más énfasis en la expedición de títulos académicos y en la adquisición de competencias laborales (Fernández Paradas, Fernández Paradas, y Gutiérrez Montoya 2017) que en el desarrollo y bienestar interno del individuo, esto conlleva no contemplar la felicidad como un elemento formativo de vital relevancia pedagógica (Elwick y Cannizzaro 2017; Guilherme y De Freitas 2017). En la sociedad del conocimiento esta metodología no estimula la interacción entre docente y educando, ni la creación de un clima positivo y creativo en las aulas (Karabchuk 2019), siendo ello contrario a lo que ocurre en algunas universidades anglosajonas tales 
como Stanford, Yale y Harvard, cuyos planes de estudios contemplan la ciencia de la felicidad como una asignatura transversal.

En el caso de Harvard sus alumnos de psicología del liderazgo han vivenciado que la instrucción en temas de felicidad mejora de forma considerable no solo su calidad de vida personal y psicológica sino también su rendimiento académico (Goldberg 2006). Tal hecho sirvió que los directivos de ciertas universidades de Europa y América Latina empezaran a tomar mayor conciencia sobre la importancia de implementar políticas y estrategias didácticas que contribuyan al desarrollo integral y holístico de la felicidad en el entorno académico (Alonso, Fernández-Salinero y Topa 2019; Ravina-Ripoll et. al. 2019; Schmidt y Hansson 2018). Ahora bien, no se debe olvidar que el bienestar individual de los futuros egresados está influenciado también por la historia, la cultura y las costumbres de la institución donde cursan sus carreras universitarias (Fulmer et. al. 2010).

En el contexto señalado, las instituciones de enseñanza superior de naturaleza religiosa empiezan de igual manera a ser conscientes, por un lado, de la relevancia que posee la variable religión en el constructo multidimensional del bienestar subjetivo de los estudiantes (Aghababaei y Błachnio 2014; Bayani 2014; Moltafet, Mazidi y Sadati 2010; Alavi 2007), y por otro, que un sistema de aprendizaje colaborativo orientado en enriquecer las competencias interpersonales, las habilidades sociales, la inteligencia emocional y la creatividad incrementarán de forma significativa la felicidad individual de los futuros graduados en la era del conocimiento digital (Baytemir 2019; Demir et. al. 2012; Pannells y Claxton 2008).

Bajo esta perspectiva y en virtud de todo lo expuesto anteriormente, los autores de este artículo se plantearon dentro del marco de la Universidad 4.0, la siguiente pregunta ¿Deben las universidades estimular la generación de proyectos docentes que tengan como principal finalidad la puesta en marcha de acciones pedagógicas enfocadas a cultivar íntegramente la felicidad cognitiva de sus estudiantes? (Correa y Moreira 2019; Caballero-García y Sánchez-Ruíz 2018). Con el objeto de poder responder a dicha cuestión se debe tener en cuenta que las investigaciones previas señalan que una didáctica sustentada en contenidos y sistemas de evaluación tradicionales incrementan el nivel de estrés académico y por ende afectan sustantivamente el bienestar psicológico de los educandos (Preoteasa, Axante, Cristea y Preoteasa 2016), y que los universitarios más felices:

- Suelen poseer grandes probabilidades de finalizar con éxito su formación académica (Frisch, et. al. 2005). 
- Tienen mayores oportunidades en la era del Big Data de acceder a un puesto de trabajo estable y de calidad, el cual les proporcionará altas cotas de satisfacción laboral y status económico (Staw, Sutton y Pelled 1994).

- Poseen un elevado engagement académico en su vida estudiantil (Datu, King y Valdez 2017).

En este sentido, los resultados alcanzados por los trabajos científicos de Bailey y Phillips (2016), Saffari et. al. (2014), Ramírez y Fuentes (2013) y Martínez Martínez et. al. (2005) demuestran empíricamente la existencia de sólidas relaciones positivas entre las variables felicidad y rendimiento académico en el ámbito universitario. Sin embargo, hay escasas investigaciones encaminadas a realizar análisis comparativos sobre dicho constructo, ante lo cual el presente trabajo plantea las siguientes hipótesis de estudio:

- H1. Cuanto mayor sea la percepción de la felicidad de los estudiantes de la Universidad de Cádiz mejor será su rendimiento académico.

- H2 Cuanto mayor sea la percepción de la felicidad de los estudiantes de la Universidad Autónoma de Baja California mejor será su rendimiento académico.

- H3: El nivel del impacto de la felicidad sobre el rendimiento académico entre estudiantes mexicanos y españoles es diferente en los grupos de estudio.

La naturaleza de las hipótesis determina que al tener el mismo resultado las primeras dos se rechaza la tercera, y en caso de existir diferencia entre ellas, la tercera hipótesis se acepta. Se presentan los posibles resultados ilustrados en la Tabla 1.

Tabla 1. Determinación de posibles resultados en evaluación de hipótesis

\begin{tabular}{|c|c|c|}
\hline H1 (F - $>$ RA | España ) & H2 (F - > RA | México) & H3 (H1 $<$ H2) \\
\hline V & V & F \\
\hline V & F & V \\
\hline F & V & F \\
\hline F & F & V \\
\hline
\end{tabular}

$\mathrm{F}=$ Felicidad y $\mathrm{RA}=$ Rendimiento Académico 


\section{METODOLOGÍA}

En esta investigación de corte cuantitativo, aleatorio, no experimental y correlacional, los datos fueron recogidos a través de una herramienta web para aplicar cuestionarios. Para este efecto se utilizó una encuesta estructurada a partir de la literatura académica existente sobre el tópico abordado. La población de la muestra de esta investigación lo conforman los 207 estudiantes del curso académico 2018-2019, 143 alumnos para el caso de España de la Facultad de Ciencias Económicas y Empresariales de la Universidad de Cádiz (UCA), y 64 estudiante en el de México los de la Facultad de Contaduría y Administración de la Universidad Autónoma de Baja California (UABC). Con respecto a la variable sexo, 106 son mujeres y 101 son hombres, no encontrándose diferencias significativas en la distribución de sexo entre ambas instituciones de enseñanza superior. Ahora bien, conviene señalar que en la universidad española se detenta una mayor presencia de féminas y en la universidad mexicana la relación es inversa, presentándose más hombres, como se observa en la Tabla 2.

Tabla 2. Perfil demográfico de la muestra

\begin{tabular}{ccccccc}
\hline $\begin{array}{c}\text { Univer- } \\
\text { sidad }\end{array}$ & Muestra & Hombres & Mujeres & $\begin{array}{c}\text { Edad Mí- } \\
\text { nima }\end{array}$ & $\begin{array}{c}\text { Edad } \\
\text { Máxima }\end{array}$ & $\begin{array}{c}\text { Edad } \\
\text { Media }\end{array}$ \\
\hline UCA & 143 & 66 & 77 & 18 & 62 & 22,99 \\
UABC & 64 & 35 & 29 & 18 & 42 & 22,69 \\
Total & 207 & 101 & 106 & 18 & 62 & 22,90
\end{tabular}

En ellas se detecta no solo que las edades de los estudiantes están comprendidas entre los 18 y 62 años sino además que la edad máxima se produce en la Universidad de Cádiz con un registro de 62 años. En cuanto a la edad media se aprecia que la institución superior de enseñanza española posee una tasa más elevada con respecto a la universidad de México, exactamente en 0,21 puntos. Una vez mostrado el perfil demográfico de los alumnos entrevistados, se expone seguidamente la ficha de la encuesta empleada en el desarrollo del presente trabajo. 
Tabla 3. Ficha técnica de la encuesta

\begin{tabular}{ll}
\hline Unidad de análisis & $\begin{array}{l}\text { Estudiantes de Tecnologías, Administración y Direc- } \\
\text { ción de Empresa, Contaduría y Negocios Internacio- } \\
\text { nales }\end{array}$ \\
\hline Ámbito geográfico & $\begin{array}{l}\text { Universidad de Cádiz-Campus de Cádiz (España) y } \\
\text { Universidad Autónoma de Baja California - Campus } \\
\text { Tijuana (México) }\end{array}$ \\
\hline Universo de la población & $\begin{array}{l}5.190 \text { estudiantes matriculados en el periodo 2018 - } \\
2019\end{array}$ \\
\hline Tipo de muestreo & Por conveniencia \\
\hline Tamaño de la muestra & 207 \\
\hline Error muestral & $5,6 \%$ \\
\hline Nivel de confianza & $90 \%$ \\
\hline Fecha de realización & Septiembre 2018 - Febrero 2019 \\
\hline Tipo de pregunta & Enunciado afirmativo \\
\hline Escala & Likert de 1 a 7 puntos \\
\hline Número de ítems & 14 [8 - rendimiento académico; 6 - felicidad] \\
\hline
\end{tabular}

En la Tabla 3 se observan las características principales que definen la muestra del sujeto de investigación. En este caso, la aplicación del instrumento de medición se ha realizado a estudiantes universitarios de la Universidad de Cádiz y de la Universidad Autónoma de Baja California durante el periodo escolar de 2018-2019. Así mismo, el proceso de obtención de la información se realizó por conveniencia y está definida con un tamaño de la muestra de 207 individuos, con un error muestral de 5,6\% y un nivel de confianza al $90 \%$.

El desarrollo de los cuestionamientos obedece al modelo conceptual ilustrado en la Figura 1 siguiente. En este sentido, se elaboraron 14 ítems para cada variable, siendo 6 para la variable de felicidad y 8 para la variable de rendimiento académico. La escala usada en este instrumento fue la de Likert con valores de 1 a 7 puntos de valoración de percepción, recorriendo de Totalmente en Desacuerdo para el menor a Totalmente de Acuerdo para el mayor. La composición de la estructura de cada ítem está caracterizada por un enunciado positivo que el respondiente valora utilizando la escala mencionada. Un ejemplo de ítem es "Me siento feliz en mi entorno académico", con lo que ilustra la estructura mencionada de enunciado positivo para su evaluación. 


\section{MODELO CONCEPTUAL}

El modelo por medio del cual se elabora la presente investigación corresponde a la estructuración de las hipótesis que surgen de las preguntas de investigación sobre la influencia de la felicidad en el rendimiento académico. Es a través de los constructos que forman parte de la variable compuesta que se desarrolla en la Figura 1. Y que se refieren a la presunción de un impacto significativo en el rendimiento escolar en dos muestras ubicadas en locaciones geográficamente distantes con diferencias sociales, económicas, políticas y culturales. La hipótesis $\mathrm{H} 3$ asume que existe una percepción diferente entre ambos grupos, por lo que se espera que al menos una hipótesis planteada sea rechazada.

Figura 1. Modelo Conceptual del Proceso de Investigación

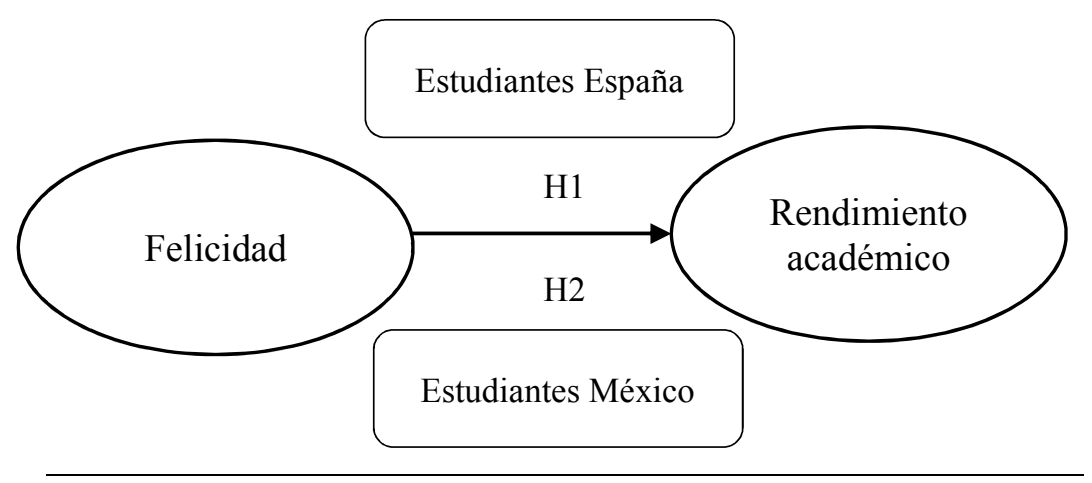

$\mathrm{H} 3: \mathrm{H} 1<>\mathrm{H} 2$

\section{RESULTADOS}

\section{PRUEBA DE CONFIABILIDAD}

En el instrumento utilizado se puede observar que al realizar el análisis de confiabilidad de los resultados de la aplicación del cuestionario se obtienen elevados índices de alfa de Cronbach, esto indica que la confiabilidad del instrumento es significativa, datos que se reflejan en la Tabla 3. Con la finalidad de conocer la percepción de la felicidad de los educandos de estas dos instituciones de enseñanza superior se aplicó un cuestionario integrado por ocho ítems para la variable rendimiento académico y seis ítems para la variable felicidad. A partir de esta rica fuente de información se empleó el coeficiente Alpha de Cronbach. Dicho indicador estadístico se emplea para verificar que los cuestionamientos aplicados midan efectivamente lo aspectos que se pretende observar o investigar. Según Nunally (1967), el coeficiente Alpha de Cronbach debe dar 
una cifra superior a 0,6 puntos para demostrar la confiabilidad en la escala usada que en este instrumento fue la escala de Likert con valores de 1 a 7 puntos, recorriendo la percepción de Totalmente en Desacuerdo para el menor y Totalmente de Acuerdo para el mayor.

Tabla 4. Estudio de Confiabilidad-Alpha de Cronbach

\begin{tabular}{lllll}
\hline No. & Variable & Ítems & UCA & UABC \\
\hline 1 & $\begin{array}{l}\text { Rendimiento } \\
\text { Académico }\end{array}$ & 8 & 0,814 & 0,803 \\
\hline 2 & Felicidad & 6 & 0,890 & 0,856 \\
\hline
\end{tabular}

De acuerdo con la información que figura en la Tabla 4, se aplicó este instrumento cuantitativo para las variables felicidad y rendimiento académico. En la primera de ella se contempla nítidamente que los valores alcanzados por los estudiantes de la Universidad Cádiz $(0,890)$ así como por los de la Universidad Autónoma de Baja California $(0,856)$ sobrepasan dicha prueba de forma bastante satisfactoria. En el caso del segundo parámetro, es decir, la percepción del rendimiento académico de nuestra población muestral, se pone de manifiesto que los índices de Alpha de Cronbach para los estudiantes de la Universidad Cádiz $(0,814)$ y los de la Universidad Autónoma de Baja California $(0,803)$ están también por encima del registro de 0,6 puntos. Ambos valores garantizan que este instrumento estadístico es apto para medir el fenómeno objeto de la presente investigación.

\section{CORRELACIÓN DE PEARSON}

A continuación, se procede a describir de forma generalista las correlaciones que existen entre los parámetros felicidad y el rendimiento académico como variables independiente y dependiente respectivamente en este estudio. 
Tabla 5. Correlación de Pearson-Estudiantes de España-UCA

\begin{tabular}{|c|c|c|c|}
\hline & & Felicidad & $\begin{array}{l}\text { Rendimiento } \\
\text { Académico }\end{array}$ \\
\hline \multirow{3}{*}{ Felicidad } & Correlación de Pearson & 1 & \\
\hline & Sig. (bilateral) & & \\
\hline & $\mathrm{N}$ & 143 & \\
\hline \multirow{3}{*}{$\begin{array}{l}\text { Rendimiento } \\
\text { Académico }\end{array}$} & Correlación de Pearson & $0,506^{* *}$ & 1 \\
\hline & Sig. (bilateral) & 0,000 & \\
\hline & $\mathrm{N}$ & 143 & 143 \\
\hline
\end{tabular}

**. La correlación es significativa al nivel 0,01 (bilateral).

En la Tabla 5 se detecta una alta correlación entre las variables felicidad y rendimiento académico $(\mathrm{r}=0,506$ y $\mathrm{p}<0.001)$. Esta interpretación no implica causalidad; la relación significa solamente que las variables covarían entre ellas por lo que si existe relación mutua.

Tabla 6. Correlación de Pearson-Estudiantes de México-UABC

\begin{tabular}{llcc}
\hline & & $\mathrm{F}$ & $\mathrm{RA}$ \\
\hline \multirow{3}{*}{ Felicidad } & \multicolumn{1}{l}{ Correlación de Pearson } & 1 & \\
\cline { 2 - 4 } & \multicolumn{1}{l}{ Sig. (bilateral) } & 64 & \\
\cline { 2 - 4 } & $\mathrm{N}$ & $0,491^{* *}$ & 1 \\
\hline \multirow{2}{*}{$\begin{array}{l}\text { Rendimiento } \\
\text { Académico }\end{array}$} & Correlación de Pearson & 0,000 & \\
\cline { 2 - 4 } & Sig. (bilateral) & 64 & 64 \\
\cline { 2 - 4 } & $\mathrm{N}$ &
\end{tabular}

**. La correlación es significativa al nivel 0,01 (bilateral).

Así mismo, en los datos ilustrados en la Tabla 6 se muestra una alta correlación entre los parámetros felicidad y rendimiento académico $(\mathrm{r}=0,491$ y $\mathrm{p}<$ 0.001). Dicha interpretación no conlleva causalidad; la relación significa solamente que las variables covarían entre ellas por lo que si existe relación mutua.

Tabla 7. Correlación de Pearson-Evaluación de Hipótesis

\begin{tabular}{ccccc}
\hline Hipótesis & Descripción & $\mathrm{r}$ & $\mathrm{p}$ & Conclusiones \\
\hline $\mathrm{H} 1$ & $\mathrm{~F}->$ RA en España & $\mathrm{r}=0,506$ & 0,000 & Se acepta \\
\hline H2 & F -> RA en México & $\mathrm{r}=0,491$ & 0,000 & Se acepta \\
\hline H3 & H1 $>$ H2 & & & Se rechaza \\
\hline
\end{tabular}


Con el fin de concentrar la información obtenida en el estudio de correlaciones de ambas muestras, se concentra la información ilustrada en la Tabla 7 que reflejan las percepciones de los individuos en el universo de estudio entre estudiantes universitarios de la Universidad Cádiz y los de la Universidad Autónoma de Baja California. En los resultados de ambas muestras se detecta la existencia de una relación relevante entre la variable felicidad como factor que impacta en el rendimiento académico Este hallazgo está en sintonía con las hipótesis $\mathrm{H} 1$ y H2. Esto significa dos cosas, por un lado, que se aceptan ambas hipótesis, y por otro lado, que la hipótesis H3 se rechace obedeciendo a su naturaleza comparativa ilustrada en la Tabla 1.

\section{ANOVA DE UN FACTOR}

A continuación, se lleva a cabo el análisis ANOVA para encontrar la relación entre la variable predictora felicidad y la variable dependiente rendimiento académico obteniendo los resultados que se presentan en las Tablas 8 y 9 .

Tabla 8. ANOVA de un factor-Estudiantes de España-UCA

\begin{tabular}{lccccc}
\hline & Suma de cuadrados & df & Media cuadrática & F & Sig. \\
\hline Intragrupos & 3044,288 & 25 & 121,772 & 3,191 & 0,000 \\
\hline Entregrupos & 4465,377 & 117 & 38,166 & & \\
\hline Total & 7509,664 & 142 & & & \\
\hline
\end{tabular}

En la Tabla 8 se descubre una relación positiva entre el rendimiento académico y la felicidad para el caso de los estudiantes de la Universidad de Cádiz. En este sentido, se advierte la conveniencia de que la percepción de felicidad se debe impulsar y mejorar en la medida de lo posible para impactar sobre el rendimiento académico. Los resultados del análisis de varianza indican que la variable predictora posee un impacto significativo sobre el rendimiento académico. Asimismo los datos de felicidad, donde $F(25,117)=3,191$ y $p=0.000$, presenta una tendencia hacia el incremento de rendimiento académico conforme se acreciente la percepción del ratio felicidad. 
Tabla 9. ANOVA de un factor-Estudiantes de México- UABC

\begin{tabular}{lccccc}
\hline & Suma de cuadrados & df & Media cuadrática & F & Sig. \\
\hline Intragrupos & 873,193 & 19 & 45,958 & 1,489 & 0,137 \\
\hline Entregrupos & 1358,167 & 44 & 30,867 & & \\
\hline Total & 2231,359 & 63 & & & \\
\hline
\end{tabular}

En la Tabla 9 se detenta que no hay una relación directa entre el rendimiento académico y felicidad para los universitarios mexicanos. Por tanto, se puede afirmar grosso modo que la percepción de felicidad en este grupo de individuos no influye positivamente sobre el rendimiento académico. Los resultados del análisis de varianza señalan que la variable predictora no tiene un gran impacto sobre el rendimiento académico. Los datos de felicidad, donde $\mathrm{F}(19,44)=1,489$ y $\mathrm{p}=0.137$, no evidencian una tendencia de incremento sobre el rendimiento académico conforme a la percepción subjetiva de la felicidad .

Tabla 10. ANOVA de un factor-Evaluación de Hipótesis

\begin{tabular}{|c|c|c|c|c|}
\hline Hipótesis & Descripción & $\mathrm{F}$ & $\mathrm{p}$ & $\begin{array}{c}\text { Conclusio- } \\
\text { nes }\end{array}$ \\
\hline $\mathrm{H} 1$ & F -> RA en España & $\mathrm{F}(25,117)=3,191$ & 0,000 & Se acepta \\
\hline $\mathrm{H} 2$ & F -> RA en México & $\mathrm{F}(19,44)=1,489$ & 0,137 & Se rechaza \\
\hline H3 & $\mathrm{H} 1<>\mathrm{H} 2$ & & & Se acepta \\
\hline
\end{tabular}

En los datos ilustrados en la Tabla 10 se advierte que los estudiantes españoles manifiestan valores que corroboran la hipótesis H1 planteada en el presente artículo. Por otra parte, los futuros graduados mexicanos expresan que el concepto de variable felicidad no es un factor que impacte sobre su rendimiento académico como se ha sugerido en la hipótesis H2. Dicho resultado origina que la hipótesis $\mathrm{H} 3$ se acepte obedeciendo a su naturaleza comparativa ilustrada en la Tabla 1, ello deja entrever que existen diferencias en la percepción de felicidad según el territorio donde se efectúe los estudios universitarios. 


\section{CONCLUSIONES Y DISCUSIONES}

En esta investigación se aplicaron un total de 207 cuestionarios, de los cuales 64 son de la Universidad Autónoma de Baja California en México y 143 de la Universidad de Cádiz en España. Las variables objetos del presente estudio se analizaron por medio de las técnicas estadísticas del Alfa de Cronbach, correlación de Pearson y la ANOVA. Los hallazgos obtenidos ponen de manifiesto los siguientes aspectos:

- La hipótesis H1 que indica que a mayor felicidad habrá mejor rendimiento académico en estudiantes de la Universidad de Cádiz se valida en las pruebas estadísticas de correlación de Pearson y la ANOVA. Esto confirma de manera dual la relevancia de la felicidad en el desarrollo cognitivo de los futuros graduados (Ravina-Ripoll et. al. 2019; Ahumada-Tello 2017).

- La hipótesis H2 que hace referencia a la felicidad como factor de impacto en el rendimiento académico de los estudiantes de la Universidad Autónoma de Baja California se ratifica en la prueba de correlación de Pearson, pero no así en la técnica ANOVA, por esta razón se rechaza y como resultado final de la investigación se considera que no se cumplen los requisitos para su aceptación aunque en el ejercicio de correlación haya sido aceptada.

- La hipótesis H3 que se enfoca en un análisis comparativo entre las hipótesis $\mathrm{H} 1$ y $\mathrm{H} 2$ nos y muestra dos cosas básicamente. La primera que la prueba de correlación de Pearson la rechaza, y la segunda que el instrumento empírico ANOVA la acepta, la naturaleza de $\mathrm{H} 3$ se encuentra descrita en la Tabla 1 y se establece que es a partir de evaluar los resultados de $\mathrm{H} 1$ y $\mathrm{H} 2$ que se llega a su aceptación o rechazo, en este caso, al final del proceso de investigación se rechaza por evidencia en una de las dos pruebas estadísticas llevadas a cabo. Esta disyuntiva debe animarnos a efectuar futuros trabajos de investigación que refuerzan o no la hipótesis planteada a lo largo de estas páginas.

Los resultados encontrados confirman la necesidad de ampliar el espectro de variables a atender para comprender el fenómeno educativo de incrementar el rendimiento académico. Como se puede confirmar, la felicidad si es importante para uno de los grupos investigados sin embargo, las diferencias encontradas en las pruebas de ANOVA, indican que existen otros factores por descubrir. Es por ello que en trabajos futuros es conveniente realizar abordajes con características holísticas, por ejemplo incluir las diferencias en sistemas educativos, 
las cuestiones económicos, sociales y culturales, la influencia de las tendencias modernas en cuanto a equidad de género y modificación o consolidación de valores individuales y sociales.

Este tema de estudio es amplio y de igual manera es importante para comprender el comportamiento hacia la consecución de un éxito académico. Se espera que el rendimiento académico sea un antecedente de lo que en el futuro será la capacidad de resolución de problemas y cumplimiento de objetivos en el ámbito laboral. Inclusive ir más allá y considerar que las conductas tendientes hacia buenos resultados se puede extrapolar al éxito en las relaciones personales.

\section{REFERENCIAS BIBLIOGRÁFICAS}

Aghababaei, N., y Błachnio, A. (2014). Purpose in life mediates the relationship Aghababaei, Naser, and Agata Błachnio. 2014. "Purpose In Life Mediates The Relationship Between Religiosity And Happiness: Evidence From Poland". Mental Health, Religion \& Culture 17 (8): 827-831. doi:10.1080/1367 4676.2014.928850.

Ahumada Tello, Eduardo. 2017. "Percepción De Competitividad Personal. Un Estudio Desde La Perspectiva De La Felicidad, El Bienestar Y La Educación En Egresados Universitarios". Revista De Estudios Empresariales. Segunda Época, no. 1: 34-54. doi:10.17561/ree.v0i1.3187.

Ahumada-Tello, Eduardo, J.J. Hernández G de Velasco, and Rafael RavinaRipoll. 2018. "El Rol De Las Redes Digitales En El Desempeño Académico Y En La Percepción De Felicidad Social En Estudiantes Universitarios En Baja California (2017)". In Claves Para Un Desarrollo Sostenible La Creatividad Y El Happiness Management Como Portafolio De La Innovación Tecnológica, Empresarial Y Marketing Social, 1st ed., 99-116. Granada, España: Editorial Comares.

Alarcón, Reynaldo. 2006. "Desarrollo De Una Escala Factorial Para Medir La Felicidad". Interamerican Journal Of Psychology 40 (1): 96-106. http://www. redalyc.org/articulo.oa?id=28440110.

Alavi, Hamid Reza. 2007. "Correlatives Of Happiness In The University Students Of Iran (A Religious Approach)". Journal Of Religion And Health 46 (4): 480-499. doi:10.1007/s10943-007-9115-4.

Alonso, Consuelo, Samuel Fernández-Salinero, and Gabriela Topa. 2019. "The Impact Of Both Individual And Collaborative Job Crafting On Spanish Teachers' Well-Being”. Education Sciences 9 (2): 74-83. doi:10.3390/educsc i900074. 
Alvarez Pertuz, Alicia Amalia, Omar de Jesús Sandoval Fernández, Janeth del Carmen Saker García, and Gloria Cecilia Moreno Gómez. 2017. Competencias Ciudadanas Para La Formación Integral En Educación Superior. 1st ed. Barranquilla, Colombia: Editorial Corporación Universidad de la Costa, EDUCOSTA.

Bailey, Thomas Hamilton, and Lisa J. Phillips. 2015. "The Influence Of Motivation And Adaptation On Students' Subjective Well-Being, Meaning In Life And Academic Performance". Higher Education Research \& Development 35 (2): 201-216. doi:10.1080/07294360.2015.1087474.

Bayani, Ali Asghar. 2014. "The Relationship Between Religiosity And Happiness Among Students In An Iranian University". Pertanika Journal Of Social Science And Humanities22 (3): 709-716. http://www.pertanika.upm.edu.my/ Pertanika\%20PAPERS/JSSH\%20Vol.\%2022\%20(3)\%20Sep.\%202014/02\%20Page\%20709716\%20(JSSH\%200861-2013\%20Short\%20Comm).pdf.

Baytemir, Kemal. 2019. "Experiences Of School As A Mediator Between Interpersonal Competence And Happiness In Adolescents". Anales De Psicología 35 (2): 259-268. doi:10.6018/analesps.35.2.320311.

Caballero García, Presentación Ángeles, and Sara Sánchez Ruiz. 2018. "La Felicidad En Estudiantes Universitarios. ¿Existen Diferencias Según Género, Edad O Elección De Estudios?". Revista Electrónica Interuniversitaria De Formación Del Profesorado 21 (3): 1-18. doi:10.6018/reifop.21.3.336721.

Chan, Grace, Paul W. Miller, and MoonJoong Tcha. 2005. "Happiness In University Education". International Review Of Economics Education 4 (1): 20-45. doi:10.1016/s1477-3880(15)30139-0.

Charles-Lejia, Humberto, Gilberto Aboites, and Ignacio Llamas. 2018. "Una Revisión De Aportaciones Que Contribuyeron Al Estudio De La Utilidad Y La Felicidad En La Economía". Análisis Económico XXXIV (86): 57-76. http://www.analisiseconomico.azc.uam.mx/index.php/rae/article/view/277.

Correa, J.C., and Y.A. Moreira. 2019. "La Práctica Universitaria Inclusiva De La Universidad Metropolitana De Ecuador: Una Alternativa Desde El Bienestar Estudiantil". Revista Metropolitana De Ciencias Aplicadas 2 (1): 166-173. http://remca.umet.edu.ec/index.php/REMCA/article/view/106.

Datu, Jesus Alfonso D., Ronnel B. King, and Jana Patricia M. Valdez. 2017. "The Academic Rewards Of Socially-Oriented Happiness: Interdependent Happiness Promotes Academic Engagement". Journal Of School Psychology 61: 19-31. doi:10.1016/j.jsp.2016.12.004. 
del Valle, Macarena Verónica, and Sebastián Urquijo. 2015. "Relaciones De Las Estrategias De Codificación Mnésica Y La Capacidad De Aprendizaje Con El Desempeño Académico De Estudiantes Universitarios". Psicología Educativa 21 (1): 27-37. doi:10.1016/j.pse.2015.02.004.

Demir, Melikşah, Jas Jaafar, Nicholas Bilyk, and Mohammad Raduan Mohd Ariff. 2012. "Social Skills, Friendship And Happiness: A Cross-Cultural Investigation". The Journal Of Social Psychology 152 (3): 379-385. doi:10.1080/002 24545.2011.591451.

Easterbrook, Matthew J., Toon Kuppens, and Antony S. R. Manstead. 2015. "The Education Effect: Higher Educational Qualifications Are Robustly Associated With Beneficial Personal And Socio-Political Outcomes". Social Indicators Research 126 (3): 1261-1298. doi:10.1007/s11205-015-0946-1.

Elwick, Alex, and Sara Cannizzaro. 2017. "Happiness In Higher Education". Higher Education Quarterly 71 (2): 204-219. doi:10.1111/hequ.12121.

Fernández Paradas, R., M. Fernández Paradas, and G.A. Gutiérrez Montoya. 2017. Educación Histórica, Patrimonios Olvidados Y Felicidad En La Didáctica De Las Ciencias Sociales. San Salvador: Universidad Don Bosco.

Flynn, Deborah M., and Stephanie MacLeod. 2015. "Determinants Of Happiness In Undergraduate University Students". College Student Journal 49 (3): 452 460. https://eric.ed.gov/?id=EJ1095449.

Frey, Bruno S., and Alois Stutzer. 2002. "What Can Economists Learn From Happiness Research?". Journal Of Economic Literature XL: 402-435. https://www.bsfrey.ch/articles/_376_2002.pdf.

Frisch, Michael B., Michelle P. Clark, Steven V. Rouse, M. David Rudd, Jennifer K. Paweleck, Andrew Greenstone, and David A. Kopplin. 2005. "Predictive And Treatment Validity Of Life Satisfaction And The Quality Of Life Inventory". Assessment 12 (1): 66-78. doi:10.1177/1073191104268006.

Fulmer, C. Ashley, Michele J. Gelfand, Arie W. Kruglanski, Chu Kim-Prieto, Ed Diener, Antonio Pierro, and E. Tory Higgins. 2010. "On "Feeling Right" In Cultural Contexts: How Person-Culture Match Affects Self-Esteem And Subjective Well-Being". Psychological Science 21 (11): 1563-1569. doi:10.1177/0956797610384742.

Goldberg, Carey. 2006. "Harvard's Crowded Course To Happiness 'Positive Psychology' Draws Students In Droves". The Boston Globe, , 2006. https://archive.boston.com/news/education/higher/articles/2006/03/10/harvards_crowded_course_to_happiness/. 
Guilherme, Alex, and Ana Lucia Souza de Freitas. 2016. “'Happiness Education': A Pedagogical-Political Commitment". Policy Futures In Education 15 (1): 6-19. doi:10.1177/1478210316637489.

Jongbloed, Janine. 2018. "Higher Education For Happiness? Investigating The Impact Of Education On The Hedonic And Eudaimonic Well-Being Of Europeans". European Educational Research Journal 17 (5): 733-754. doi:10.1177/1474904118770818.

Karabchuk, Tatiana. 2019. "Interview With Tatiana Karabchuk. International Comparisons, Social Impacts Of Labor Instability, And The Secrets Of Academic Happiness". Journal Of Economic Sociology 20 (1): 12-19. doi:10.17323/1726-3247-2019-1-12-19.

Kindelán, Ma. Paz. 2013. "Una Perspectiva Sobre El Binomio Enseñanza-Investigación En La Universidad Del S. XXI". Revista Complutense De Educación 24 (1): $27-45$.

Lyubomirsky, Sonja, Chris Tkach, and Robin DiMatteo. 2006. "What Are The Differences Between Happiness And Self-Esteem". Social Indicators Research 78 (3): 363-404. doi:DOI 10.1007/s11205-005-0213-y.

Martin, Brian. 2011. "On Being A Happy Academic". Australian Universities' Review 53 (1): 50-56.

Martínez Martínez, Isabel Ma., Edgar Bresó Esteve, Susana Llorens Gumbau, and Rosa Grau Gumbau. 2005. "Bienestar Psicológico En Estudiantes Universitarios: Facilitadores Y Obstaculizadores Del Desempeño Académico". Anales De Psicologia/Annals Of Psychology 21 (1): 170-180. https://revistas.um.es/analesps/article/view/27261.

Martínez, Isabel M., Isabella Meneghel, and Jonathan Peñalver. 2019. “¿El Género Afecta En Las Estrategias De Afrontamiento Para Mejorar El Bienestar Y El Desempeño Académico?". Revista De Psicodidáctica 24 (2): 111-119. doi:10.1016/j.psicod.2019.01.003.

Moltafet, Ghavam., Mohammed. Mazidi, and Somayeh Sadati. 2010. "Personality Traits, Religious Orientation And Happiness". Procedia - Social And Behavioral Sciences 9: 63-69. doi:10.1016/j.sbspro.2010.12.116.

Moreno, Yaiza, and Rosario J. Marrero. 2015. "Optimismo Y Autoestima Como Predicadores De Bienestar Personal: Diferencias De Género". Revista Mexicana De Psicologia 32 (1): 27-36. http://www.redalyc.org/articulo.oa?id=24 3045363004.

Nikolaev, Boris, and Pavel Rusakov. 2015. "Education And Happiness: An Alternative Hypothesis". Applied Economics Letters 23 (12): 827-830. doi:10.1080/13504851.2015.1111982. 
Noddings, N. 2003. Happiness And Education. Nueva York: Cambridge University Press.

Nunnally, Jum C. 1978. Psychometric Theory. New York: McGraw-Hill Book.

Öztemel, Kemal, and Elvan Y1ldiz-Akyol. 2019. "The Predictive Role Of Happiness, Social Support, And Future Time Orientation In Career Adapta-bility". Journal Of Career Development. doi:10.1177/0894845319840437.

Pannells, T.C., and A.F. Claxton. 2008. "Felicidad, Ideación Creativa Y Locus De Control”. Revista De Investigación De La Creatividad 20 (1): 67-71.

Paschoal, Tatiane, Cláudio V. Torres, and Juliana Barreiros Porto. 2019. "Felicidade No Trabalho: Relações Com Suporte Organizacional E Suporte Social". Revista De Administração Contemporânea 14 (6): 1054-1072. doi:http://dx.doi.org/10.1590/S1415-65552010000700005.

Paso-Niebla, J., and L. Hernández-Guzmán. 2007. "Variables Que Inciden En El Rendimiento Académico De Adolescentes Mexicanos". Revista Latinoamericana De Psicología 39 (3): 487-501.

Pedroza Flores, René. 2018. "La Universidad 4.0 Con Currículo Inteligente 1.0 En La Cuarta Revolución Industrial / The University 4.0 With Intelligent Curriculum 1.0 In The Fourth Industrial Revolution". RIDE Revista Iberoamericana Para La Investigación Y El Desarrollo Educativo 9 (17): 168-194. doi:10.23913/ride.v9i17.377.

Pérez Muñoz, S., and R. Castaño Calle. 2016. "Funciones De La Universidad En El Siglo XXI: Humanística, Básica E Integral”. Revista Electrónica Interuniversitaria De Formación Del Profesorado 19 (1): 191-199.

Posada, R., and O. Aguilar. 2012. "La Felicidad Como Elemento Del Desarrollo Sostenible Desde La Organización”. Revista Del Centro De Investigación 10 (37): 83-94.

Preoteasa, Cristina Teodora, Anca Axante, Anca Diana Cristea, and Elena Preoteasa. 2016. "The Relationship Between Positive Well-Being And Academic Assessment: Results From A Prospective Study On Dental Students". Education Research International 2016: 1-8. doi:10.1155/2016/9024687.

Ramírez, Patricio E, and Carolina A Fuentes. 2013. "Felicidad Y Rendimiento Académico: Efecto Moderador De La Felicidad Sobre Indicadores De Selección Y Rendimiento Académico De Alumnos De Ingeniería Comercial”. Formación Universitaria 6 (3): 21-30. doi:10.4067/s0718-500620130003 00004.

Ravina-Ripoll, Rafael, Estela Núñez-Barriopedro, Araceli Galiano-Coronil, and Luis Tobar-Pesantez. 2019. "Towards A Happy, Creative And Social Higher Education Instituion: The Case Of Non-Profit Marketing And Business Creation Subjects At The University Of Cadiz". Journal Of Entrepreneurship 
Education 22 (1): 1-8. https://www.abacademies.org/articles/towards-ahappy-creative-and-social-higher-education-institution-the-case-of-nonprofit-marketing-and-business-creation-subjects-at-7708.html.

Saffari, Mohsen, Hornoz Sanaeinasab, Hojat Rashidi Jahan, Gholamhossein Purtaghi, and Amir Pakpour. 2014. "Happiness, Self-Efficacy And Academic Achievement Among Students Of Baqiyatallah University Of Medical Sciences". Journal Of Medical Education Development 7 (13): 45-56. $\mathrm{http}: / /$ zums.ac.ir/edujournal/article-1-185-en.html.

\section{Rafael Ravina-Ripoll}

Facultad de Ciencias Económicas y Empresariales Universidad de Cádiz. Avenida Enrique Villegas Vélez, 2 11002 Cádiz (España) https://orcid.org/0000-0001-7007-3123

Eduardo Ahumada-Tello

Facultad de Contaduría y Administración Universidad Autónoma de Baja California Calzada Universitaria 14418, Mesa de Otay 22309, Tijuana, B.C. (México) https://orcid.org/0000-0003-1698-5126 Autor de correspondencia: eahumada@uabc.edu.mx
E. Julián Gálvez-Albarracín Facultad de Ciencias de la Administración Universidad del Valle Sede San Fernando Calle 4B No. 36-00 25360 Cali (Colombia) https://orcid.org/0000-0003-3972-2310 